\title{
INCIVILIDADE NO PROCESSO DE TRABALHO DA ENFERMAGEM
}

Benedita Gonçales de Assis Ribeiro ${ }^{1}$ Sergio Valverde Marques dos Santos ${ }^{2}$ Sebastião Elias da Silveira ${ }^{3}$ Vanessa Augusto Bardaquim ${ }^{3}$ Marcia Andrade Queiroz Ozanam ${ }^{3}$ Maria Lúcia do Carmo Cruz Robazzi ${ }^{3}$

\author{
https://orcid.org/0000-0001-5587-1098 \\ https://orcid.org/0000-0001-9412-9515 \\ https://orcid.org/0000-0001-8779-8171 \\ https://orcid.org/0000-0003-2179-552X \\ https://orcid.org/0000-0003-3897-0304 \\ https://orcid.org/0000-0003-2364-5787
}

Objetivo: discutir sobre a incivilidade no processo de trabalho dos profissionais de enfermagem. Para isso, foram elaborados pontos norteadores para apoiar a reflexão do estudo, sendo eles: a civilidade e a incivilidade no trabalho e a incivilidade no trabalho da enfermagem. Desenvolvimento: A incivilidade não é um comportamento recente na enfermagem, é denominada como uma hostilidade ou violência horizontal, descrita como comportamento hostil, agressivo, por um enfermeiro ou grupo de enfermeiros, em direção a outro enfermeiro ou a um grupo, por meio de disputas internas, atitudes como rudeza ao falar com colega, resposta sarcástica, desrespeito às confidências e à privacidade. Conclusão: o comportamento incivil faz parte da rotina diária dos profissionais de enfermagem, causando prejuizo tanto para os trabalhadores como para a instituição de saúde. Torna-se importante que esse assunto seja amplamente discutido nas instituições, com foco na redução da incivilidade entre os enfermeiros e demais profissionais da saúde.

Descritores: Incivilidade; Trabalho; Enfermagem; Hospital; Saúde do trabalhador.

\section{INCIVILITY IN THE NURSING WORK PROCESS}

Objective: To discuss the incivility in the work process of nursing professionals. For this, guiding points were elaborated to support the reflection of the study, namely: civility and work incivility and nursing work incivility. Development: Incivility is not a recent behavior in nursing, it is termed as horizontal hostility or violence, described as hostile, aggressive behavior by one nurse or group of nurses toward another nurse or group through disputes. internal, attitudes such as rudeness in talking with colleague, sarcastic response, disrespect for confidentiality and privacy. Conclusion: Incivil behavior is part of the daily routine of nursing professionals, causing harm to both workers and the health institution. It is important that this issue be widely discussed in institutions, focusing on reducing incivility among nurses and other health professionals.

Descriptors: Incivility; Job; Nursing; Hospital; Worker's health.

\section{INCIVILIDAD EN EL PROCESO DE TRABAJO DE ENFERMERÍA}

Objetivo: Discutir la incivilidad en el proceso de trabajo de los profesionales de enfermería. Para esto, se elaboraron puntos de guía para apoyar la reflexión del estudio, a saber: civilidad e incivilidad laboral e incivilidad laboral de enfermería. Desarrollo: La incivilidad no es un comportamiento reciente en enfermería, se denomina hostilidad o violencia horizontal, descrita como comportamiento hostily agresivo por una enfermera o grupo de enfermeras hacia otra enfermera o grupo a través de disputas. internas, actitudes como la grosería al hablar con un colega, la respuesta sarcástica, la falta de respeto a la confidencialidad y la privacidad. Conclusión: el comportamiento incivil es parte de la rutina diaria de los profesionales de enfermería, causando daño tanto a los trabajadores como a la institución de salud. Es importante que este tema sea ampliamente discutido en las instituciones, enfocándose en reducir la incivilidad entre las enfermeras y otros profesionales de la salud.

Descriptores: Incivilidad; Trabajo; Enfermería; Hospital; Salud del trabajador.

IUniversidade Estadual de Londrina - UEL, PR

2Universidade do Estado de Minas Gerais - UEMG, MG

${ }^{3}$ Universidade de São Paulo - USP, Ribeirão Preto, SP

Autor Correspondente: Sergio Valverde Marques dos Santos Email: sergiovalverdemarques@hotmail.com

Recebido 05/12/2019 Aceito 02/04/2020 


\section{INTRODUÇÃO}

Os avanços tecnológicos trouxeram diversos benefícios para a população, no entanto, tem causado a redução da qualidade de vida e da saúde de trabalhadores. Isto, devido as modificações do mundo globalizado, que têm ocasionado mudanças no estilo de vida e no ambiente de trabalho das pessoas $^{(1)}$.

Com isso, as atividades laborais são executadas de maneira forçada e sobrecarregada, gerando insatisfação e favorecendo a ocorrência de doenças nos ambientes laborais. Fatores contemporâneos como jornadas incessantes, ambiente hostil e estressante, rodizios frequentes de turnos de trabalho, dentre outros, que estão presentes nos ambientes hospitalares são que tem afetado o trabalhador, entre eles, os profissionais de enfermagem, com impactado nas condições físicas e mentais ${ }^{(2-3)}$.

O processo de trabalho dos enfermeiros possui caracteristicas que agrupam fatores que podem causar danos à saúde física e mental. As longas jornadas de trabalho, trabalho noturno, posturas inadequadas, exposição às radiações, manuseio de produtos químicos, exposição às doenças, desgaste físico e mental, remuneração inadequada, falta de prestígio social, entre outros, são fatores que estão constantemente presentes na vida laboral desses trabalhadores e que podem favorecer um comportamento incivil(4).

A incivilidade no local de trabalho é percebida por meio de manifestações de ação rude, falta de respeito para com os outros no local de trabalho e também pelo desrespeito das regras, pelo comportamento descortês, o oposto do comportamento civilizado(5)

Normalmente, a incivilidade é dirigida a um colega de trabalho, que pode ser alguém com menos poder em relação à uma pessoa em situação de poder. Este tipo de comportamento incide, também, sobre profissionais recém-formados e, ainda, sobre os graduandos de enfermagem, por serem considerados mais suscetíveis, principalmente por serem mais jovens, com menor experiência de vida pessoal e profissional e por serem mais inibidos para o confronto ${ }^{(6-7)}$.

Os comportamentos incivis que mais têm aparecido entre os enfermeiros envolvem gritar com o colega, criticar, espalhar boatos sobre ele, faltar-lhe com o apoio. Estes comportamentos diferem de bullying e assédio moral, pois além dessas características anteriormente citadas, é acompanhado de intimidação e abuso psicológico, os quais ocorrem de forma repetitiva ${ }^{(7-8)}$.

No ambiente hospitalar, os profissionais de enfermagem estão em constantes trocas de relações interpessoais. Estas relações, muitas vezes, são cheias de sentimentos que chegam ao sofrimento, pelas situações vivenciadas, mani- festadas com raiva, humilhação, vergonha, tristeza, angústia, ansiedade, não valorização do trabalho, depressão e descontentamento laboral(9).

A incivilidade é "cara", dispendiosa para os locais onde elas acontecem e poucas são as instituições que a reconhecem e apresentam medidas para minimizá-las ou redimi-las e, ainda, que a incivilidade tem repercussões sobre a produtividade e, também, sobre o custo da perda de produtividade ${ }^{(10)}$

Frente ao exposto, percebe-se que o comportamento incivil é real nas instituições de saúde, principalmente no ambiente laboral dos enfermeiros. Entretanto, nota-se ainda uma limitação de pesquisas sobre esse tema no Brasil, assim como na literatura internacional ${ }^{(7-8)}$. Diante disso, justifica-se a realização deste estudo, devido a insuficiência de conhecimento nacional sobre esta temática, evidenciando uma lacuna no conhecimento sobre o assunto e a sua originalidade no país.

Com isso, neste estudo objetivou-se discutir sobre a incivilidade no processo de trabalho dos profissionais de enfermagem, por meio de um ensaio teórico e reflexivo sobre esta abordagem. Para isso, foram elaborados pontos norteadores para apoiar a reflexão do estudo, sendo eles: civilidade e incivilidade no trabalho e incivilidade no trabalho da enfermagem.

\section{DESENVOLVIMENTO}

\section{Civilidade e incivilidade no trabalho}

A palavra civilidade origina-se do idioma latino civile, designando o habitante da cidade - civitate. Com a agregação dos seres humanos e diante do raciocínio e consciência do Eu, iniciaram-se as relações humanas, tornando-se necessário haver o respeito entre as pessoas, surgindo a codificação das regras de convivio social(11).

O entendimento de civilidade é proporcional à ética, à modernidade, à renovação, à educação; se uma pessoa apresenta como prerrogativas um comportamento civil, ela necessita ser cordial e bem educada, tanto nas ações quanto no comportamento. O conceito de pessoa moral aplica-se quando ela compõe uma coletividade; nesse sentido a moral harmoniza-se com a ética e o respeito, elementos básicos para se ter um grupo civilizado(11).

Ser civil significa ter um comportamento que demonstra cortesia, sensibilidade e consideração pelos outros; no local de trabalho, ajuda a preservar as normas do respeito mútuo e torna-se essencial para o desenvolvimento de confiança, empatia e de relações interpessoais positivas ${ }^{(12)}$.

Em uma comunidade e no trabalho, espera-se que as pessoas que sejam tratadas com algum grau de respeito $e$ cortesia. Na ausência dessas qualidades do tratamento entre as pessoas, a incivilidade prevalece. A incivilidade é uma 
palavra que tem raízes na palavra latina antiga incivilidade, a qual significa uma falta de civilidade, ou seja, de comunidade ou cidade ${ }^{(13)}$

O termo "incivilidade" refere-se às frequentes erupções de desordem, manifestações de difícil identificação, mescladas no cotidiano, sendo compreendida, como as transgressões no cotidiano, violações do código de boas maneiras. Não são consideradas ações ilegais, mas desrespeito à ordem determinada na vida cotidiana. Assim, as expressões de incivilidades dão-se por quebras de convívio em harmonia que sinalizam uma ruptura com as normas e valores sociais convencionais. No entanto essa ruptura, trazida pelas incivilidades, vai além das regras, atingindo também as expectativas em relação à convivência e os pactos sociais que perpassam as relações humanas, questões que, se supõe, sejam aprendidas desde a infância(14).

A incivilidade é considerada uma descortesia deliberada em direção ao outro, definida como um ato de agressão, tal como, abuso verbal ou emocional ou ainda físico, sutil ou real, de um colega para com os outros. É um tipo de mau trato, uma atitude rude como ignorar alguém, interromper quem está falando, creditar para si uma ideia de outro, propalar boatos e realizar a violação não intencional de normas sociais ${ }^{(15)}$.

No ambiente laboral a incivilidade é definida como um comportamento com a intenção de prejudicar o alvo, em violação de padrões de trabalho ou da consideração e do respeito pelos outros ${ }^{(16)}$. Pode ser descrita como um comportamento desagradável/insociável de baixa intensidade, pois é considerado de menor magnitude em termos de força, como outras agressões, fornece muito incomodo e inquietação para o alvo ${ }^{(17)}$.

Constitui-se em um fenômeno inerente ao ambiente social laboral e apresenta implicações importantes para o bem estar psicológico dos trabalhadores ${ }^{(18)}$. Pode se manifestar por meio de comportamentos do tipo exclusão de pessoas, fofoca, sarcasmo, hostilidade e, até, agressão, também denominado de comportamento destrutivo(19).

Com base nesse olhar, as vítimas de incivilidade mostram maior estresse no trabalho, distração cognitiva, angustia psicológica, menor satisfação laboral e, em última análise, são mais propensas para deixar a instituição(20). A diminuição da incivilidade no local de trabalho, apresenta efeitos positivos sobre a saúde mental dos trabalhadores ${ }^{(16)}$.

\section{Incivilidade no trabalho da enfermagem}

A incivilidade não é um comportamento recente na enfermagem. Ela ocorre e é denominada como uma hostilidade ou violência horizontal, descrito como comportamento hostil, agressivo, por um enfermeiro ou grupo de enfermeiros, em direção a outro enfermeiro ou a um grupo, por meio de disputas internas, com atitudes como rudeza ao falar com colega, resposta sarcástica, desrespeito às confidências e à privacidade ${ }^{(16,21)}$.

Sabe-se que os perpetradores desses comportamentos destrutivos, em geral encontram-se no topo hierárquico, são médicos, enfermeiros, gerentes de unidades, além de profissionais que estão há mais tempo no serviço, com estabilidade no emprego e experiência clínica reconhecida ${ }^{(22)}$.

Entre enfermeiros, atos de incivilidade - como os abusos verbais - parecem ser impostos mais entre os que se encontram em início de carreira, que são mais vulneráveis aos efeitos desse abuso imposto por seus próprios colegas. Outro aspecto é que em ambientes com condições de trabalho desfavoráveis, os enfermeiros que ali atuam tendem a ter atitudes de trabalho menos favoráveis, com seus colegas mais vulneráveis, facilitando a ocorrência de situações abusivas ${ }^{(23)}$.

A incivilidade no local de trabalho, relacionado ao enfermeiro, tem sido discutido constantemente nos Estados Unidos, inclusive durante a formação e em atividades práticas nas unidades hospitalares. Tem-se que enfermeiros recém-formados, recebem atos de incivilidade de supervisores ou enfermeiros já com mais tempo de trabalho ou, ainda, o enfermeiro pode ser vítima da incivilidade de um colega de igual função ${ }^{(7.21)}$.

A incivilidade entre enfermeiros pode levar à rotatividade de trabalhadores e, até, a um mal atendimento ao paciente e outras reações indesejáveis como, vergonha e constrangimento. O descontentamento com o local de trabalho pode ser um dos fatores responsáveis pela rotatividade ${ }^{(24)}$

O autor do comportamento incivil não quer diretamente prejudicar ninguém, porém tal atitude pode induzir ao assédio moral e à violência, decorrendo em graves danos ao trabalhador, com insatisfação e ineficácia no trabalho, sobretudo nos de enfermagem ${ }^{(12)}$.

Na enfermagem, a exposição dos trabalhadores à violência no trabalho tem sido vinculada aos problemas de saúde nessas pessoas, os quais resultam em danos físicos, manifestações emocionais, transtornos e distúrbios psíquicos; acresce-se que ainda influencia o desempenho do trabalhador, bem como a sua dimensão familiar e social(25). Em especial na profissão de enfermagem com suas diversas categorias de trabalhadores, os atos de incivilidade podem ser lesivos à saúde dos que são por eles afetados(26)

Evidências demonstram que a incivilidade entre enfermeiros no trabalho provoca prejuízos para os trabalhadores e organizações. Enfermeiros que se sentem desrespeitados com 
a diminuição da produtividade, tornam-se menos criativos, diminuem a qualidade e o esforço no trabalho e ocorre um aumento no absenteísmo, casos de burnout e rotatividade(10,21,27).

Em um estudo fenomenológico realizado na Coréia, com estudantes de enfermagem, com o objetivo de avaliar a natureza e experiência de incivilidade enquanto estudantes na prática clínica. Os resultados indicaram que os estudantes de enfermagem vivenciam incivilidade de diferentes perpetradores, como, funcionários, pacientes e familiares de pacientes, durante a prática clínica ${ }^{(28)}$

Em uma pesquisa que investigou a incivilidade entre enfermeiras e mães de família em três hospitais de Mashhad, Irã, constatou-se que, pelo menos, $28,8 \%$ dos enfermeiros relataram uma forma de incivilidade em seu local de trabalho por um ou dois momentos, tais como fofocas, comportamento hostil, violação de privacidade, comportamentos estes de seus superiores ${ }^{(27)}$.

Na área da saúde, sabe-se que o processo de trabalho é desenvolvido com importante interação social, envolvendo necessidades que incluem trabalhadores do setor, gestores e pacientes. Essa interação é determinada pelas condições laborais e fatores humanos que influenciam os resultados da prestação de serviços e a segurança dos pacientes. No entanto, nos dias atuais, são identificadas fraquezas de valores, atitudes, competências e comportamentos, que podem interferir na segurança das organizações de saúde e influenciar o processo de incivilidade entre os profissionais ${ }^{(22)}$

A civilidade entre os profissionais de enfermagem é um elemento importante, para que sejam alcançados resultados positivos para os pacientes. Os profissionais de enfermagem com comportamentos adequados apresentam atitudes respeitosas, deliberadas e compassivas, favorecendo a qualidade da assistência prestada aos usuários do serviço de saúde.
Para isso, torna-se necessária uma formação profissional capaz de responder às necessidades de saúde da população e os seus preceitos éticos e legais, mesmo diante de comportamentos incivis ${ }^{(29-30)}$

\section{CONSIDERAÇÕES FINAIS}

Os comportamentos incivis fazem parte da rotina diária dos profissionais de enfermagem. Este comportamento tem causado prejuizo tanto para os trabalhadores como para a instituição de saúde.

Por isso, percebe-se a necessidade de ampliar a discussão desse tema para as instituições e profissionais de saúde. Com isso, os enfermeiros, em função de gerência, podem promover melhorarias em seu local de trabalho, assim como para a qualidade do trabalho de sua equipe, por meio da interação entre grupos e profissionais oprimidos.

Além disso, faz-se necessário a aceitação das instituições de saúde a respeito da presença de incivilidade no ambiente laboral dos profissionais. Uma vez que tal comportamento, prejudica a qualidade da assistência prestada aos usuários do serviço de saúde.

\section{CONTRIBUIÇÕES DOS AUTORES:}

Benedita Gonçales de Assis Ribeiro e Maria Lúcia do Carmo Cruz Robazzi, com a concepção e projeto, participação ativa na discussão; Sérgio Valverde Marques dos Santos e Sebastião Elias da Silveira, com a redação do artigo e revisão crítica relevante do conteúdo intelectual; Vanessa Augusto Bardaquim e Marcia Andrade Queiroz Ozanam, com a revisão e aprovação final da versão a ser publicada; Maria Lúcia do Carmo Cruz Robazzi com a concordância com todos os aspectos do manuscrito em termos de veracidade ou integridade das informações.

\section{REFERÊNCIAS}

1. Almeida GFP, Ribeiro MHA, Silva MACN, Branco RCC. Pinheiro FCM, Nascimento MDSB. Patologias osteomusculares como causa de aposentadoria por invalidez em servidores públicos do municipio de São Luis, Maranhão. Rev Bras Med Trab [Internet]. 2016 [acesso 2018 jul 05];14(1):37-44. Disponivel em: http://www.rbmt.org.br/details/21/pt-BR/patologias-osteomusculares-como-causa-de-aposentadoria-por-invalidez-em-servidores-publicosdo-municipio-de-sao-luis--maranhao

2. Huebra PM, Ribeiro CA. Crescencio LNP, Sampaio RMF, Silva RMGC, Martins WSO et. al. Condições de saúde mental e fisica do trabalhador. Anais do IV Seminário Cientifico da FACIG [Internet]. 2018. [acesso 2020 fev 13]. Disponivel em: http://pensaracademico.facig.edu.br/index.php/semiariocientifico/ article/view/782

3. Porto JS, Marziale MHP. Motivos e consequências da baixa adesão às precauções padrão pela equipe de enfermagem Rev Gaúcha Enferm [Internet] 2016 [acesso 2020 fev 13];37(2):e57395. Disponivel em: http://dx.doi.org/10.1590/1983-1447.2016.02.57395

4. Versa GLGS, Matsuda LM. Satisfação profissional da equipe de enfermagem intensivista deum hospital de ensino. Rev. Enferm. UERJ [Internet]. 2014 [acesso 2018 jul 12]:22(3):409-15. Disponivel em: http://www.facenf.uerj.br/v22n3/v22n3al9.pdf

5. Azizan HM, Razlina HJ. Relationship between workplace incivility, job attitudes and Muslim religiosty personality among trade union members. Global Journal Al-Thaqafah [Internet]. 2015 [acesso 2020 fev 13]:5(2):43-51. Disponivel em: https://www.researchgate.net/publication/288827333_ Relationship_between_Workplace_Incivility_Job_Attitudes_and_Muslim_Religiosity_Personality_among_Trade_Union_Members 
6. Vessey JA, Demarco RF, Difazio R. Bullying, harassment, and horizontal violence in the nursing workforce: The state of the science. Annual Review of Nursing Research, 2011:28(1):133-57.

7. Macnamara SA. Incivility in nursing: unsafe nurse, unsafe patients. AORN Journal [Internet]. 2012 [acesso 2018 jul 20]:95(4):535-40. Disponivel em: https://aornjournal.onlinelibrary.wiley.com/doi/pdf/10.1016/j.aorn.2012.01.020

8. Clark CM, Ahten SM. Begning the conversation: the nurse educator's role in preventing incivility in the workplace. Georgia Nursing [Internet]. 2012 [acesso 2018 jul 20];72(3):16-7. Disponivel em: https://scholarworks.boisestate.edu/nursing_facpubs/87/

9. Dalmolin GL, Lunardi VL, Barlem ELD, Silveira RS. Implications of moral distress on nurses and its similarities with burnout. Texto Contexto Enferm [Internet]. 2012 [acesso 2018 jul 26]:21(1):200-8. Disponivel em: https://www.redalyc.org/pdf/714/71422299023.pdf

10. Porath C, Pearson C. The price of incivility lack of respect hurts morale - and the bottom line. Harvard Business Review. 2013;91(1/2):114-21.

11. Hollanda SB. Raizes do Brasil. 26 ed. São Paulo, SP: Companhia das Letras; 1995.

12. Pearson CM, Andersson LM, Porath CL. Workplace incivility. In: Fox S, Spector PE. (Ed.). Counterproductive work behaviour: investigations of actors and targets. 1 ed. Washington, DC: American Psychological Association; 2005.

13. Connelly RJ. Introducing a culture of civility in first-year college classes. The Journal of General Education [Internet]. 2009 [acesso 2018 Set 10];58(1):47-68. Disponivel em: https://muse.jhu.edu/article/266050/pdf

14. Garcia J. Indisciplina, incivilidade e cidadania na escola. Educ Temát Digit [Internet]. 2006 [acesso 2018 Set 10]:8(1):121-30. Disponivel em http://www. ssoar.info/ssoar/bitstream/handle/document/19764/ssoar-etd-2006-1-garcia-indisciplina.pdf?sequence=1

15. Peters AB. Faculty to faculty incivility: experiences of novice nurse faculty in academia. Journal of Professional Nursing [Internet]. 2014 [acesso 2018 Set 15];30(3):213-27. Disponivel em: https://www.sciencedirect.com/science/article/pii/S875572231300152X

16. Laschinger HKS. Wong CA. Cummings GG. Grau AL. Resonant leadership and workplace empowerment: The value of positive organizational cultures in reducing workplace incivility. Nursing Economics [Internet]. 2014 [acesso 2018 Set 20]:32(1):5-16. Disponivel em: https://search.proquest.com/openview/ alf74b4696b0a03f6lblfb55ed5e54b8/1?pq-origsite=gscholar\&cbl=30765

17. Vickers MH. Writing what' relevant: Workplace Incivility in public administration - a wolf in sheep's clothing. ATP [Internet]. 2006 [acesso 2018 Set 21];28(1):69-88. Disponivel em: https://www.tandfonline.com/doi/abs/10.1080/10841806.2006.11029525

18. Nitzsche M. (In)Civilidade no trabalho: Escalas de Medida e Efeitos no Burnout e Engagement. Dissertação. Psicologia Clínica e de Aconselhamento. [Internet]. 2016. [acesso 2020 fev 13]. Lisboa. Disponivel em: https://repositorio.ual.pt/handle/11144/2545

19. Schilpzand P. Plater IE, Erez A. Workplace incivility: a review of the literature and agenda for future research. J Organiz Behav [Internet]. 2016 [acesso 2018 Out 05]:37(S):57-88. Disponivel em: https://onlinelibrary.wiley.com/doi/full/10.1002/job.1976

20. Cortina LM, Magley VJ. Patterns and profiles of response to incivility in the workplace. Journal of Occupational Health Psychology [Internet]. 2009 [acesso 2018 Out 09];14(3):272-88. Disponivel em: https://psycnet.apa.org/record/2009-09794-005

21. Becher J, Visovsky C. Horizontal violence in nursing. Medsurg Nursing [Internet]. 2012 [acesso 2018 Out 11];21(4):210-39. Disponivel em: https://www. researchgate.net/profile/Constance_Visovsky/publication/230831024_Horizontal_violence_in_nursing/links/57ac75b508ae7a6420c2e593.pdf

22. Oliveira RM, Silva LMS, Guedes MVC, Oliveira ACS, Sánchez RG, Torres RAM. Analyzing the concept of disruptive behavior in healthcare work: an integrative review. Rev. esc. enferm. USP [Internet]. 2016 [acesso 2018 Out 11];50(4):695-704. Disponivel em: http://www.scielo.br/pdf/reeusp/ v50n4/0080-6234-reeusp-50-04-0695.pdf

23. Budin WC, Brewer CS, Chao YY, Kovner C. Verbal abuse from nurse colleagues and work environment of early career registered nurses. J Nurs Scholarsh [Internet]. 2013 [acesso 2019 Jan 10];45(3):308-16. Disponivel em: https://sigmapubs.onlinelibrary.wiley.com/doi/pdf/10.1111/jnu.12033

24. Leiter MP, Day A, Oore DG, Spence Laschinger HK. Getting better and staying better: Assessing civility, incivility, distress, and job attitudes one year after a civility intervention. Journal of Occupational Health Psychology [Internet]. 2012 [acesso 2019 Jan 10];17(4):425-34. Disponivel em: https://psycnet. apa.org/record/2012-27459-004

25. Bordignon M. Monteiro MI. Violence in the workplace in Nursing: consequences overview. Rev Bras Enferm [Internet]. 2016 [acesso 2019 Jan 20];69(5):939-42. Disponivel em: http://dx.doi.org/10.1590/0034-7167-2015-0133

26. Mammen B, Hills DJ, Lam L. Newly qualified graduate nurses' experiences of workplace incivility in Australian hospital settings. Collegian [Internet]. 2018 [acesso 2019 Jan 20]:25(6):591-9. Disponivel em: https://www.sciencedirect.com/science/article/pii/S1322769618300581

27. Heydari A, Rad M, Rad M. Evaluating the incivility between staff nurses and matrons employed in Iran. Scientific Journal of the Faculty of Medicine in Nis [Internet]. 2015 [acesso 2019 Jan 21];32(2):137-46. Disponivel em: https://www.degruyter.com/downloadpdf/j/afmnai.2015.32.issue-2/afmnai-2015-0014/ afmnai-2015-0014.pdf

28. Park KO, Kim JK. Experience of Incivility to Nursing Students during Clinical Practice. Journal of Korean Academy of Nursing Administration [Internet]. 2017 [acesso 2020 fev 13];:23(5):524. Disponivel em: https://doi.org/10.11ll1/jkana.2017.23.5.524

29. Oja KJ. Incivility and Professional Comportment in Critical Care Nurses. AACN Advanced Critical Care [Internet]. 2017 [acesso 2020 fev 14];28(4):345350. Disponivel em: https://www.ncbi.nlm.nih.gov/pubmed/29212641

30. Brito FMM, Rozendo CA, Sobral JPCP. O laboratório de enfermagem e a formação crítica do enfermeiro: uma reflexão. Enferm. Foco [Internet].2018 [acesso 2020 fev 14]:9(1): 36-40. Disponivel em: https://doi.org/10.21675/2357-707X.2018.v9.nl.1859 